\title{
Effects of kinesio taping on the gait parameters of children with cerebral palsy: a pilot study
}

\author{
Sun-Hye Jung ${ }^{a, b}$, Sun-Hae Song ${ }^{b}$, Da-Rye Kim ${ }^{c}$, Seul-Gi Kimc, Ye-Jin Park ${ }^{c}$, Yeon-Jung Son ${ }^{c}$, GyuChang Lee \\ a JungWoo Children Development Center, Changwon, Republic of Korea \\ ${ }^{b}$ Department of Physical Therapy, Graduate School of Kyungnam University, Changwon, Republic of Korea \\ 'Department of Physical Therapy, Kyungnam University, Changwon, Republic of Korea
}

Objective: Children with spastic diplegic cerebral palsy show weakness especially in the lower-extremity rather than upper-extremity muscles and display characteristics such as asymmetric alignment, deficits in postural control or balance ability, and slow walking speed. Various therapeutic interventions are applied to children with cerebral palsy, of which taping is widely used in the field of rehabilitation, however, there are few studies of the effects of kinesio taping on gait patterns of children with cerebral palsy. The present study investigated the effects of kinesio taping on gait parameters of children with cerebral palsy.

Design: Cross-sectional study.

Methods: Four children with spastic diplegia participated in this study. The participants' gait parameters while walking $10 \mathrm{~m}$ with and without kinesio taping (tibialis anterior, quadriceps femoris, and gluteus maximus) were recorded. Gait parameters including gait velocity, cadence, step length, stride length, single support time, and double support time were evaluated using the GAITRite. Mean values were obtained after having the subjects walk three times in each condition with a 5-minute rest period between each condition. The order of each condition was assigned randomly.

Results: There were significant improvements in gait velocity, step length, stride length, and single support time of the right leg with kinesio taping condition compared to the without kinesio taping condition taping $(p<0.05)$. However, there were no significant differences in cadence, single support time of the left leg, or double support time.

Conclusions: The results show that kinesio taping may have a positive effect for improving gait parameters of children with spastic diplegia. However, its usefulness in the rehabilitation of children with cerebral palsy needs to be further investigated.

Key Words: Cerebral palsy, Gait, Kinesio taping

\section{Introduction}

Recent advancements in medical technology have dramatically increased the survival rate of premature and verylow-birth-weight infants, resulting in an increase in the incidence of children with cerebral palsy [1]. Cerebral palsy is a permanent nonprogressive disease affecting the development of the brain and can be caused by preterm birth, intrauterine infection, periventricular leukomalacia or intraventricular hemorrhage, brain malformations, or head trauma caused by an accident occurring prenatally, during the process of birth, or within 2 years after birth [2]. Children with cerebral palsy in particular, have motor dysfunction. These problems are closely related to abnormal muscle tone, muscle weakness, contracture, and balance and gait [3] that inevitably result in a negative influence on independent daily life [4].

Cerebral palsy is normally categorized into spastic, ataxic, athetoid, or flaccid according to the movement aspect of children based on the degree of brain damage [5]. Of children with cerebral palsy, more than $7 \%$ are spastic [6,7], and children with spastic diplegic cerebral palsy especially show

Received: 23 November, 2016 Revised: 13 December, 2016 Accepted: 13 December, 2016

Corresponding author: GyuChang Lee

Department of Physical Therapy, Kyungnam University, 7 Gyeongnamdaehak-ro, Masanhappo-gu, Changwon 51767, Republic of Korea Tel: 82-55-249-2739 Fax: 82-55-999-2173 E-mail: leegc76@kyungnam.ac.kr

(c) This is an Open-Access article distributed under the terms of the Creative Commons Attribution Non-Commercial License (http://creativecommons.org/licens es/by-nc/4.0) which permits unrestricted non-commercial use, distribution, and reproduction in any medium, provided the original work is properly cited.

Copyright $@ 2016$ Korean Academy of Physical Therapy Rehabilitation Science 
weakness in lower-extremity rather than upper-extremity muscles and display characteristics such as asymmetric alignment, deficits in postural control or balance ability, and slow walking speed [8]. In previous studies, a meaningful difference was identified in the maximum muscular strength of knee extensor muscles between children with spastic diplegic cerebral palsy and normal children, and such a difference will influence gait $[9,10]$. Various therapeutic interventions are applied to children with cerebral palsy, of which taping is widely used in the field of rehabilitation beyond sports for the purposes of strengthening muscle, increasing flexibility, and reducing pain [11]. Kinesio tape is accessible owing to its relatively low cost and noninvasive application methods [12]. When kinesio tape is attached to skin, it lifts the skin from the muscles and creates wrinkles that create a wider space between muscles and skin, thus improving the circulation of blood and lymph fluid [13].

Although the effects of taping have been suggested by previous studies, there are few studies of the effects of kinesio taping on gait patterns of children with cerebral palsy. Thus, this study investigated changes in gait pattern according to the application of taping targeting lower-extremity muscles in children with cerebral palsy.

\section{Methods}

\section{Subjects}

Children with spastic diplegic cerebral palsy from a child development center were enrolled. The center's message board was used to recruit volunteers who were enrolled and screened. The inclusion criteria were 1) attendance at a regular school, 2) level 1-2 in the Gross Motor Function Classification System and Manual Ability Classification System, 3) able to walk independently at least $10 \mathrm{~m}$, and 4) able to understand and follow simple instructions. Exclusion criteria were 1) severe visual and hearing impairment, 2) uncontrolled seizures, 3 ) genetic or syndromic conditions, and 4) botulinum toxin injection in the lower extremities within the previous 6 months. Table 1 shows participant characteristics.

\section{Ethical considerations}

All participants and their guardians provided signed informed consent after receiving an explanation of the study's purpose and procedures. The study was approved by the Kyungnam University Institutional Review Board (1040460A-2016-006).

\section{Design and setting}

The present study was a comparative cross-sectional study. Characteristics of the participants including age, sex, height, weight, and leg length were collected through brief interviews. Following the interviews, gait parameters with and without kinesio taping of the lower extremity were recorded by research assistants. Kinesio taping was applied to each participant's tibialis anterior, rectus femoris, and gluteus maximus muscles. For the tibialis anterior muscle, the kinesio tape was attached along a line that passed the medial condyle of the ankle and the medial sole and went to the centerline of the instep with the ankle in a state of plantar flexion [14]. For the rectus femoris muscle, the tape was applied from $10 \mathrm{~cm}$ below the anterior superior iliac spine to the superior edge of the patella (without tension). The tape was then crossed from the edges of the patella (with maximum tension) and fixed below the inferior edge of the patella while the knee was flexed [15]. For the gluteus maximus muscle, two Y-shaped pieces of tape (approximately $35 \mathrm{~cm}$ long and $5 \mathrm{~cm}$ wide) were applied with the tails of the $\mathrm{Y}$ being $30 \mathrm{~cm}$ long and $2.5 \mathrm{~cm}$ wide, leaving a base of $5 \mathrm{~cm}$, which is the estimated distance between the participant's greater trochanter and fifth lumber (L5) spinous process [16] (Figure 1). In addition, the elasticity of the tape was set at $50 \%-70 \%$ of the available tension. The participants were asked to walk three times in each condition with and without kinesio taping, with a 5-minute rest between each condition. The order of conditions to be performed was assigned randomly by a researcher. Participants were asked to perform under each condition while walking a 10-m distance. Participants were shown a mark placed on the wall at eye level approximately $10 \mathrm{~m}$ away and were instructed to walk toward the mark. The participants were allowed to get plenty

Table 1. Characteristics of participants

\begin{tabular}{lc}
\hline Characteristic & Value \\
\hline Gender & 2 \\
Male & 2 \\
Female & $11.25(1.71)$ \\
Age (y) & $132.5(5.57)$ \\
Height $(\mathrm{cm})$ & $31.75(4.57)$ \\
Weight $(\mathrm{kg})$ & \\
Leg length $(\mathrm{cm})$ & $65.75(7.46)$ \\
Left & $63.25(5.68)$ \\
Right &
\end{tabular}

Values are presented as number only or mean (SD). 

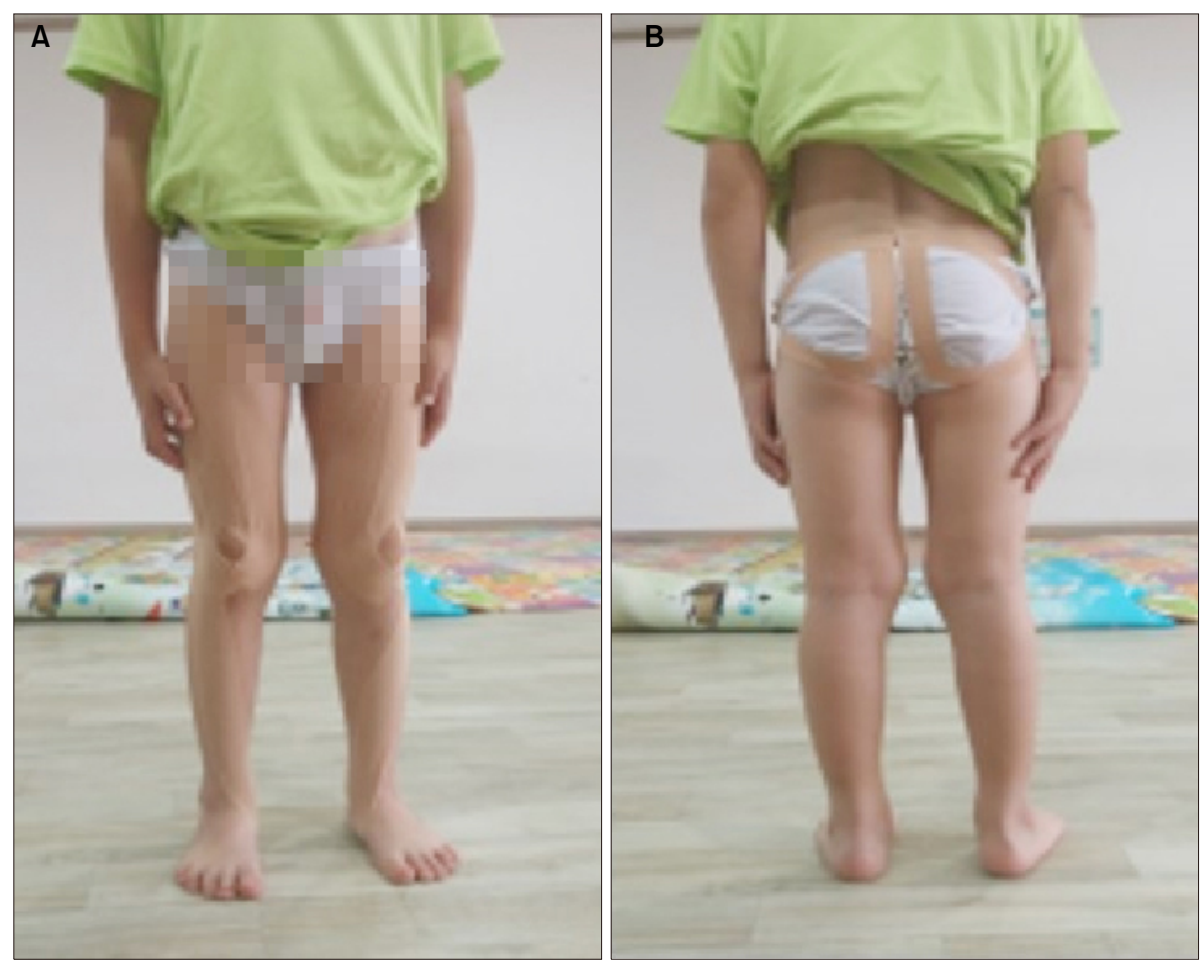

Figure 1. Kinesio taping on tibialis anterior, rectus femoris, and gluteus maximus muscles. (A) Anterior view, (B) posterior view. of rest when complaints over inconveniences were raised during the experiment. All the experiments were conducted in a quiet and spacious laboratory for physical therapy.

\section{Measurements}

The spatiotemporal parameters of gait were measured using GAITRite (GAITRite Electronic Walkway; CIR Systems Inc., Clifton, NJ, USA). Parameters including gait velocity, cadence, step length, stride length, single support time, and double support time were evaluated. The GAITRite is an electronic walkway $(4.8 \mathrm{~m}$ long and $0.9 \mathrm{~m}$ wide) that contains sensors arranged in a pattern to measure the pressure exerted by the feet on the contact surface. The device acquires data that are transmitted via connections to a laptop; application software within the computer processes patterns from the soles of the feet. The GAITRite was installed approximately $1 \mathrm{~m}$ past the starting line to reduce participants' awareness of the device. Participants walked approximately $10 \mathrm{~m}$, which included the length of the GAITRite. The test-retest reliability of the GAITRite was $0.902-0.970$, and the inter-rater reliability was $0.945-1.000$ [17].

\section{Data analysis}

For data analysis, SPSS ver. 12.0 (SPSS Inc., Chicago, IL,
USA) was used. The normality of variables was assessed by the Shapiro-Wilk test. Data on spatiotemporal parameters of the gait were analyzed by using the paired $t$-test for comparison between parameters obtained with and without kinesio taping. The level of statistical significance was set at $p$ $<0.05$.

\section{Results}

Significant differences were found between the following parameters obtained with and without kinesio taping: gait velocity, step length, stride length, and single support time of the right leg ( $p<0.05$; Table 2). However, no significant differences were found between the two conditions in cadence, single support time of left leg, and double support time (Table 2).

\section{Discussion}

The present study investigated the changes in gait variables of children with spastic diplegic cerebral palsy after applying kinesio tape on lower-extremity muscles. Walking speed, step length of both legs, and stride length were improved, and single support time was meaningfully decreased.

Chinn et al. [18] and Deschamps et al. [19] reported that 
Table 2. Comparison of gait parameters between without taping and with taping

\begin{tabular}{lccc}
\hline & $\begin{array}{c}\text { Without taping } \\
(\mathrm{n}=4)\end{array}$ & $\begin{array}{c}\text { With taping } \\
(\mathrm{n}=4)\end{array}$ & $p$ \\
\hline Velocity & $74.93(17.59)$ & $87.68(17.51)$ & 0.010 \\
Cadence & $105.10(10.92)$ & $115.75(11.87)$ & 0.073 \\
Step length (cm) & & & \\
$\quad$ Left & $43.05(7.69)$ & $45.31(7.24)$ & 0.024 \\
$\quad$ Right & $42.10(8.23)$ & $45.47(8.37)$ & 0.023 \\
Stride length (cm) & & & \\
$\quad$ Left & $85.16(15.46)$ & $91.04(14.46)$ & 0.006 \\
$\quad$ Right & $85.88(15.14)$ & $91.20(14.60)$ & 0.005 \\
Single support time (sec) & & & \\
$\quad$ Left & $0.39(0.02)$ & $0.35(0.03)$ & 0.097 \\
$\quad$ Right & $0.41(0.07)$ & $0.39(0.05)$ & 0.005 \\
Double support time (sec) & & & \\
$\quad$ Left & $0.33(0.06)$ & $0.30(0.06)$ & 0.061 \\
$\quad$ Right & $0.34(0.07)$ & $0.30(0.07)$ & 0.063 \\
\hline
\end{tabular}

Values are presented as mean (SD).

taping applied to patients with chronic ankle instability significantly improved stability of the ankle joint and gait. According to Boguszewski et al. [20], taping applied to healthy adults significantly augmented the activation and strength of lower-extremity muscles. Furthermore, Ekiz et al. [15] and Kim et al. [12] found an increase in walking speed after taping of stroke patients in a 10-meter walking test. Thus, previous studies have shown that taping improved muscle strength and functional movement along with ankle stability and muscle activation.

This study also found that taping on lower-extremity muscles resulted in a meaningful improvement in gait velocity, step length, stride length, and single support time of the right leg in children with spastic diplegic cerebral palsy. Children with spastic diplegic cerebral palsy display asymmetrical alignment due to weakness of the lower-extremity muscles $[8,17]$. Normally, because of weakness of the gluteus maximus muscle during the stance phase, anterior tilt of the pelvis, flexion-adduction of the hip, and internal rotation of the joint occur. Furthermore, owing to weakness of the quadriceps femoris muscle, the flexion of the knee joint becomes augmented. In addition to that, during the swing phase, owing to weakness of the tibialis anterior muscle, children with cerebral palsy show a gait pattern of asymmetric steps such as hyper-plantar flexion-eversion of the ankle. Previous studies analyzing the effects of lower-extremity taping on dynamic balance and gait ability in stroke patients showed meaningful differences in dynamic balance, gait velocity, and cadence, which may have resulted from improvement in the stability of the joint on which the taping was applied [21]. This study also found that taping applied on the tibialis anterior, quadriceps femoris, and gluteus maximus muscles of children with cerebral palsy positively contributed to improvement in the activation of the corresponding muscles and the stability of body posture resulting in a significant improvement of gait variables including gait velocity, step length, stride length, and single support time of the right leg.

A limitation of this study is the small number of only four participants, making it difficult to generalize the results. In addition, regarding the selection of muscles on which taping was applied, this study focused on muscles involved in flexion of the hip joint according to foot drop, and did not include muscles involved in full extension of the knee joint and hyper-anterior pelvic tilt, which also affect the gait of children with cerebral palsy. Taping was not attached to the gluteus medius, gluteus minimus, hamstring and iliopsoas muscles, even though they also influence gait.

However, this study showed that walking speed, step length, and stride length were meaningfully improved and right single support time was significantly reduced so that gait ability was increased by taping on lower-extremity muscles in children with spastic diplegic cerebral palsy. Such results suggest the possibility of temporary effects of taping to improve the gait ability of children with spastic diplegic cerebral palsy. Further studies regarding the application and intervention of taping to supplement the asymmetric gait pattern of children with cerebral palsy would be worthwhile.

\section{Conflict of Interest}

The authors declared no potential conflicts of interest with respect to the authorship and/or publication of this article.

\section{References}

1. Khandaker G, Smithers-Sheedy H, Islam J, Alam M, Jung J, Novak I, et al. Bangladesh Cerebral Palsy Register (BCPR): a pilot study to develop a national cerebral palsy $(\mathrm{CP})$ register with surveillance of children for CP. BMC Neurol 2015;15:173.

2. Nelson KB. Can we prevent cerebral palsy? N Engl J Med 2003; 349:1765-9.

3. Papavasiliou AS. Management of motor problems in cerebral palsy: a critical update for the clinician. Eur J Paediatr Neurol 2009;13:387-96.

4. Pirpiris M, Wilkinson AJ, Rodda J, Nguyen TC, Baker RJ, Nattrass GR, et al. Walking speed in children and young adults 
with neuromuscular disease: comparison between two assessment methods. J Pediatr Orthop 2003;23:302-7.

5. Rethlefsen SA, Ryan DD, Kay RM. Classification systems in cerebral palsy. Orthop Clin North Am 2010;41:457-67.

6. Nelson KB. The epidemiology of cerebral palsy in term infants. Ment Retard Dev Disabil Res Rev 2002;8:146-50.

7. Reddihough D. Cerebral palsy in childhood. Aust Fam Physician 2011;40:192-6.

8. Goh HT, Thompson M, Huang WB, Schafer S. Relationships among measures of knee musculoskeletal impairments, gross motor function, and walking efficiency in children with cerebral palsy. Pediatr Phys Ther 2006;18:253-61.

9. Thompson N, Stebbins J, Seniorou M, Newham D. Muscle strength and walking ability in diplegic cerebral palsy: implications for assessment and management. Gait Posture 2011;33: 321-5.

10. Aisen ML, Kerkovich D, Mast J, Mulroy S, Wren TA, Kay RM, et al. Cerebral palsy: clinical care and neurological rehabilitation. Lancet Neurol 2011;10:844-52.

11. Lewis JS, Wright C, Green A. Subacromial impingement syndrome: the effect of changing posture on shoulder range of movement. J Orthop Sports Phys Ther 2005;35:72-87.

12. Kim WI, Choi YK, Lee JH, Park YH. The effect of muscle facilitation using kinesio taping on walking and balance of stroke patients. J Phys Ther Sci 2014;26:1831-4.

13. Kase K, Wallis J, Kase T. Clinical therapeutic applications of the Kinesio Taping method. 2nd ed. Tokyo: Ken Ikai Co Ltd.; 2003.

14. Lee K, Yi CW, Lee S. The effects of kinesiology taping therapy on degenerative knee arthritis patients' pain, function, and joint range of motion. J Phys Ther Sci 2016;28:63-6.

15. Ekiz T, Aslan MD, Özgirgin N. Effects of Kinesio Tape application to quadriceps muscles on isokinetic muscle strength, gait, and functional parameters in patients with stroke. J Rehabil Res Dev 2015;52:323-31

16. Mostert-Wentzel K, Swart JJ, Masenyetse LJ, Sihlali BH, Cilliers R, Clarke L, et al. Effect of kinesio taping on explosive muscle power of gluteus maximus of male athletes. South Afr J Sport Med 2012;24:75-80.

17. Woollacott MH, Shumway-Cook A. Postural dysfunction during standing and walking in children with cerebral palsy: what are the underlying problems and what new therapies might improve balance? Neural Plast 2005;12:211-9.

18. Chinn L, Dicharry J, Hart JM, Saliba S, Wilder R, Hertel J. Gait kinematics after taping in participants with chronic ankle instability. J Athl Train 2014;49:322-30.

19. Deschamps K, Dingenen B, Pans F, Van Bavel I, Matricali GA, Staes F. Effect of taping on foot kinematics in persons with chronic ankle instability. J Sci Med Sport 2016;19:541-6.

20. Boguszewski D, Tomaszewska I, Adamczyk JG, Białoszewski D. Evaluation of effectiveness of kinesiology taping as an adjunct to rehabilitation following anterior cruciate ligament reconstruction. Preliminary report. Ortop Traumatol Rehabil 2013; 15:469-78.

21. Hyun KH, Cho HY, Lim CG. The effect of knee joint Mulligan taping on balance and gait in subacute stroke patients. J Phys Ther Sci 2015;27:3545-7. 